\title{
La "reputación online" de la información de vacunas en internet
}

\author{
Ubaldo Cuesta CAmbra \\ Universidad Complutense de Madrid \\ ucuesta@ucm.es \\ Sandra GASPAR HERRERo \\ Universidad Complutense de Madrid \\ sgasparherrero@ucm.es
}

\begin{abstract}
Resumen:
El objetivo de esta investigación ${ }^{1}$ ha sido: 1) identificar webs, blogs y prensa digital en las que tratan sobre vacunas y construir una taxonomía 2) monitorizar diariamente el discurso analizando variables cualitativas y cuantitativas 3 ) desarrollar un análisis cuantitativo y cualitativo de los debates en términos de la "Teoría del Frame" (Entman, 1993). Los resultados permiten obtener una clasificación precisa y objetiva del discurso social contemporáneo sobre vacunas, conociendo los temas, canales, etc. más relevantes, confirmando el empleo de estereotipos negativos por parte de determinados emisores de baja repercusión social (antivacunas) frente a un planteamiento riguroso mucho más frecuente.
\end{abstract}

Palabras clave: Comunicación anti vacunas; Comunicación y Salud; Redes sociales; salud 2.0

\section{The "online reputation" of immunization information online}

\begin{abstract}
:
The aim of this research was to: 1) identify sites, blogs and digital media in dealing with vaccines and build a taxonomy 2) monitor daily the speech analyzing qualitative and quantitative variables 3) to develop a quantitative and qualitative discussions terms of the "Theory of Frame" (Entman, 1993). The results allow obtaining a precise and objective classification of contemporary social discourse on vaccines, knowing the issues, canals, etc.. most relevant, confirming the use of negative stereotypes by certain issuers of low social impact (anti-vaccine) versus a more rigorous approach prevalent.
\end{abstract}

Key Words: anti-vaccine communication; health communication; social networks; health 2.0

Referencia normalizada:

Cuesta Cambra, U. y Gaspar Herrero, S. (2014): La "reputación online" de la información de vacunas en internet. Historia y Comunicación Social. Vol. 19. Núm. Especial Febrero. Págs. 15-29.

Sumario: 1.Introducción. 2. Objetivos. 3. Planteamiento de Research Questions. 4. Metodología. 4.1. Procedimiento. 4.2. Instrumentos. 4.3. Muestra. 5. Resultados. 5.1. Canales de comunicación. 5.2. topics sobre vacunas. 5.3. Autor. 5.4. Valoración de los autores sobre el topic. 5.5. Comentarios de los participantes. 5.6. Rigor de los datos. 6. Conclusiones. 7. Bibliografía. 


\section{Introducción}

Internet supuso un gran cambio en la manera de acceder a la información por parte de los usuarios en temas de salud para su asesoramiento en contenidos o en toma de decisiones, como puede ser la vacunación o no-vacunación, de la propia persona (Chanel, Luchini, Massoni, Vergnaud 2011) o su administración en niños (Downs, de Bruin, Fischhoff, 2008).

Gran parte de las enfermedades existentes (y algunas ya erradicadas) han sido controladas gracias al proceso de vacunación de las personas, tal y como se ha demostrado a lo largo del tiempo en diversos estudios científicos (Ehreth, 2003; World Health Organization, 2008), pero aún sigue existiendo cierta desconfianza en su aplicación en determinados sectores (Breiman, 2001).

En el caso concreto de las vacunas, aunque la ciencia está claramente a favor de su vacunación en la mayoría de los casos. Existen ciertos riesgos y preguntas que las personas desean resolver al tomar la decisión de vacunarse o no (Gust, Darling, Kennedy, Schwartz, 2008).

La aparición de las redes sociales, en el contexto de la vacunación, sumado a la "especificidad" de la problemática vinculada a las mismas, provocan dudas sobre la eficacia de los modelos tradicionales de persuasión y motivación del mensaje de salud pública empleado habitualmente. Se sugiere que los nuevos modelos de comunicación de decisión informada puede que sean más efectivos para explicar las conductas comunicativas mediatizadas a través de la web (Witterman, Zikmund-Fisher, 2011), de lo que han venido siendo los modelos tradicionales.

Los efectos producidos por las redes sociales en la influencia de las mentes de los internautas, pueden llegar a conformar determinados procesos de decisión, que suponen grandes implicaciones para las decisiones estratégicas de comunicación sobre las vacunas.

Existen tres características que definen la web 2.0: 1) participación de los usuarios, 2) actitud receptiva y 3 ) efectos de la network. Las implicaciones de estas características son:

(1) Cualquier persona que disponga de tiempo, tenga el deseo y el acceso tecnológico pertinente puede variar fuentes de recursos online sobre vacunación, (2) la gente puede acceder a bases de datos reenviando recursos y fuentes ya existentes, o construyendo nuevos recursos y (3) las personas pueden conectarse y colaborar con otros individuos con ideas similares y de ese modo, mejorar y enriquecer la participación de otros y de las fuentes de recursos existentes.

Internet y los "social media" (en especial las redes sociales), se han convertido actualmente en una poderosa herramienta para acceder a información sobre temas de salud. Concretamente, la prensa digital, blogs, webs de asociaciones y privadas son las herramientas más empleadas por los usuarios para comprobar información 
de actualidad relacionada con vacunas, y su reputación en la red (Bodemer, Muller, Okan, García-Retamero, Neumeyer-Gromen, 2012).

Existe una discusión abierta sobre los riesgos y beneficios de la vacunación; sin embargo, estos debates científicos, basados en evidencias, se observan en menor medida en los debates online. En este tipo de debates de la blogosfera, es frecuente que, debido a la polarización de la naturaleza del debate, se tienda a terminar por simplificar los mensajes excesivamente. Observar las ideas conflictivas puede llevar a las personas a dar contra-sentidos a la información y reducir su credibilidad (Wathen, Burkell, 2002).

Además, se ha observado frecuentemente que los contenidos, sites y grupos de interés respecto a las vacunas, presentan ciertas peculiaridades y son diferentes al resto de los campos de la salud. Se trata sobre medicina preventiva contra enfermedades que pueden haber sido una vez prevalentes pero que ahora raramente lo son. Es imposible identificar los individuos que evitan la enfermedad a través de la vacunación; no hay una comunidad online natural de aquellos que recogen los beneficios de las vacunas, sin embargo la web 2.0 facilita la congregación de todos aquellos que "creen" que las vacunas son dañinas.

Base a todo lo anterior, resultaría de gran utilidad conocer los sites donde actualmente se producen flujos de información sobre vacunas, qué tipo de información se emite y cuándo se provocan debates y la naturaleza de los mismos.

\section{Objetivos}

El objetivo general de esta investigación consiste en detectar, clasificar y estudiar la reputación online de los sites donde aparezca información de vacunas. Los objetivos específicos de la investigación son:

1. Analizar toda la información sobre vacunas presente en la red mediante monitorización activa, para conocer los sites y poder construir una taxonomía de los mismos (tipos de web, blog, prensa digital...).

2. Monitorización diaria (tracking continuo) del discurso analizando las variables cualitativas y cuantitativas pertinentes: canales de comunicación, topics sobre vacunas, autores de las noticias, valoraciones sobre los topics, comentarios de los participantes y carácter tipo negativo o positivo, y rigor de los datos.

3. Realización de un análisis cualitativo de los debates en términos de la "Teoría del Frame" (Entman, 1993) para conocer cómo es el discurso de estos debates $\mathrm{y}$, especialmente, de los emisores anti vacunas y contrastar si el tipo de información aportada es seria o no; es decir, rigurosa y de calidad científica basada en evidencias, o anecdótica y basada en especulaciones. 


\section{Planteamiento y desarrollo de Research Questions}

La credibilidad y la valoración de la información online de salud es un proceso complejo que puede incorporar múltiples factores que interactúan entre sí como la identificación con la fuente, el "framing", el orden, la coherencia y presentación visual del mensaje, habilidades individuales y del receptor con respecto a la literatura existente, habilidad con los números (estadísticas), e incluso factores como la elección del momento oportuno "timing” del mensaje ( Wathen, Burkell, 2002).

La búsqueda de información sobre temas relacionados con la salud en internet es cada vez más importante, resultando en muchos casos indispensable para la toma de decisiones por parte de los usuarios, en concreto en el caso de la vacunación. La primera Research Question que se plantea es:

RQ1: De forma objetiva, medida y cuantificada: ¿se puede: establecer una identificación y clasificación sobre los sites en los que aparece información sobre vacunas?

En las redes sociales aparecen narrativas e historias personales, que se convierten en modelos de expectativas donde se desarrollan, con alto nivel de detalle, las conductas realizadas por los internautas. Debido a la gran cantidad de información existente en internet y la diversidad de usuarios, se plantea la segunda Research Question:

RQ2: ¿La información de los sites, podría clasificarse en función de determinadas variables taxonómicas? Adicionalmente, ¿esta clasificación permitiría definir si dicha información es rigurosa y científica?

La información aportada en los debates puede provocar fuertes influencias sobre las reglas de decisión informada que van creándose los usuarios de esas redes. Esto supone grandes implicaciones para las decisiones estratégicas de comunicación sobre las vacunas. Es importante conocer, por lo tanto, el número y la importancia y la "calidad" de dichos debates.

RQ3: ¿Los debates que aparecen son relevantes? ¿Son rigurosos o, por el contrario, aportan información anecdótica y valorativa?

\section{Metodología}

\subsection{Procedimiento}

Se realizó una monitorización previa de la blogosfera con el objetivo de conocer los sites donde aparecía información sobre el tema. Posteriormente se fijaron alertas en motores como Google Alerts. Después, se realizó una monitorización diaria de noticias aparecidas en sites (web, blogs y prensa digital) durante periodo de tiempo 
de once meses: de diciembre de 2011 a noviembre de 2012.Dos codificadores previamente entrenados realizaron dicha monitorización.

Por otro lado, se realizó un análisis cuantitativo y cualitativo del discurso.

Para la monitorización se empleó el uso de motores de búsqueda (Mayer, Leis, 2009) para buscar archivos almacenados en servidores web y facilitar el hallazgo de nuevas páginas con contenidos relacionados con vacunas y los debates que allí se encuentran. Algunos de los motores de búsquedas más empleados: Wasalive, Socialmention, Addictomatic, Google (Google Alerts, Google News, Google academic, Google Blogs, Google Reader), Tweet Reach y Bing.

Se realizaron búsquedas en los motores con las keywords "vacunas", "vacunación España", "vacuna gripe", "antivacunas", para garantizar la calidad de los resultados y la continuidad del procedimiento llevado a cabo en la investigación. Se clasificaron los resultados de las búsquedas en una base de datos con los blogs y canales de información anti vacunas.

\subsection{Instrumentos}

Se emplearon plantillas para la codificación de los datos cualitativos y cuantitativos, para la realización del análisis diario de la información sobre vacunas. Así mismo se analizó el discurso social mediante la "Teoría del Frame" (Etman, 1993). Se utilizaron tres tipos de plantillas:

1. Plantilla codificación de temas: contiene el resumen del tema (noticia analizada); fuente (link exacto de la noticia); lugar y fecha donde acontece la noticia; tipo de vacuna del que se habla (papiloma virus, gripe, movimientos anti vacunas, otros); autor de la noticia (sanitario, periodista, otro); canal de información (prensa digital, blog o web); listado de temas (¿sobre qué se habla?, política, recomendación de vacunas, farmacéuticas, movimientos anti vacunas, nuevos brotes de enfermedades, otros; valoración del autor sobre el tópico (Negativa, neutra, buena); rigor de los datos (anecdótico o científico); y el resumen cualitativo del discurso: dividido en cuatro variables: ¿Quién dice qué? ¿A Quién? ¿Por qué Canal? ¿Y con qué efectos?

2. Plantilla análisis de debates: en el caso de que la noticia tuviera algún tipo de debate sobre el tema, se analizó en función de las siguientes variables: tipo de dominio (lugar donde fue encontrada la noticia); fuente (link exacto de la noticia); nombre del debate generado, resumen del tema, resumen de las discusiones (porcentaje de comentarios a favor, en contra, neutrales, numero de comentarios otros).

3. Plantilla análisis cualitativo (análisis del Frame): resumen del tema, las causas (razones que generan el problema), consecuencias (de la noticia o tema), buenos y malos (personajes que intervienen dentro de la noticia y si ejercen influencia de forma positiva o negativa), y las posibles soluciones que se pueden aportar al conflicto. 


\subsection{Muestra}

La muestra estaba compuesta por un total de 100 medios analizados dentro de prensa digital y de 20 webs y blogs, los cuales tras analizar los mensajes, se dividieron en diferentes canales de comunicación:

1. Oficiales: Instituciones, organizaciones, asociaciones de profesionales, académicas o científicas (Como Organizaciones sin fines de lucro).

2. No oficiales: (blogs, dominios o personas que no estén respaldadas por ningún ente externo como asociaciones, son personas particulares. Éstas a su vez serán divididas en dos grupos.

- Serias: informan sobre vacunas, donde se genera debate o no. Sus fundamentos pueden ser anecdóticos o científicos y el fin último es informar a los internautas

- Catastrofistas: de carácter anti-vacuna (ofrecen información y respuestas a comentarios con un origen no científico, provocando alarma social y desinformación.

\section{Resultados}

Los resultados obtenidos de la investigación se analizan en diferentes apartados:

\subsection{Canales de comunicación}

Los canales de comunicación representan los sitios web donde se ha encontrado la noticia, bien sea prensa digital general, prensa digital de salud, blog personal, blog privados, web privada o web de asociación. El canal donde se obtiene la mayor cantidad de información sobre vacunas es prensa digital. Los más representativos han sido "europapress.es", "noticias.lainformación.com" y "abc.es". Representa un $80 \%$ del total de los canales de comunicación estudiados con un total de 537 noticias.

Los Blogs personales representan el 6\% (44 noticias). La Web privada obtiene un $5 \%$ lo que corresponde a 32 noticias. Los Blog privados poseen un $4 \%$ con un total de 27 noticias. La Prensa digital de Salud representa el $3 \%$ del total con 14 noticias. Por último, las de web asociaciones (asociaciones de salud, consejerías, sociedades entre otros) agrupan un $2 \%$ lo que corresponde a 9 noticias. 
1. Canales de comunicación

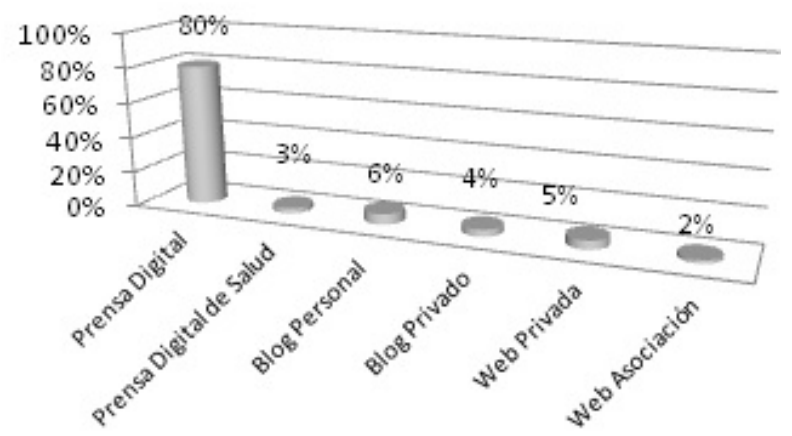

Fuente: Elaboración propia.

\subsection{Topics sobre vacunas}

Los topics que se indican a continuación corresponden a los 5 más importantes de la investigación, el resto de temas tienen un índice bajo y poco significativo dentro de las temáticas aparecidas (se detallan en gráfico a continuación):

- Programas de salud pública 35\% (326 noticias).

- Información general de vacunas 13\% (129 noticias).

- Nuevas vacunas 8\% (71 noticias).

- Nuevos brotes de enfermedades 7\% (68 noticias).

- Recomendación de vacunas 6\% (58 noticias).

\section{Topics sobre vacunas}

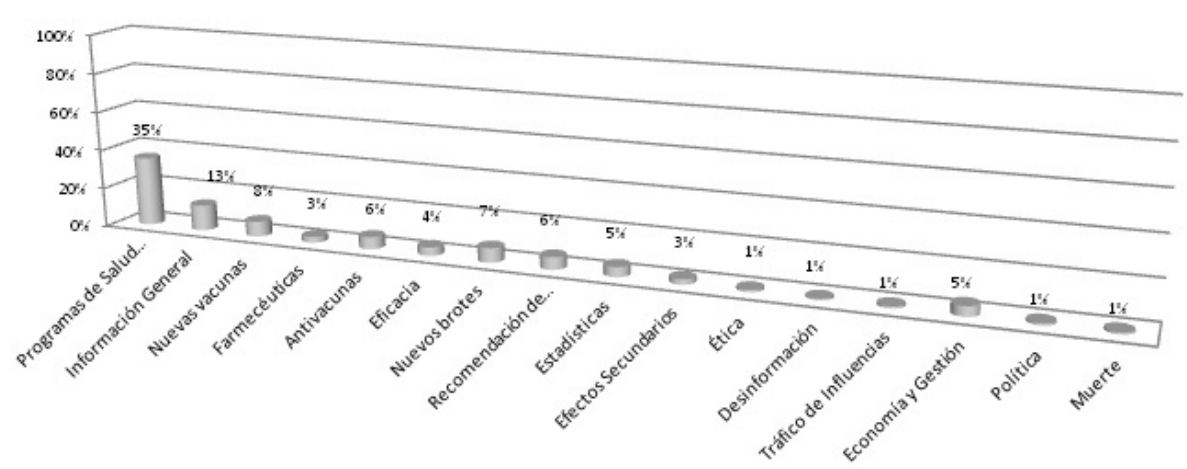

Fuente: Elaboración propia. 


\subsection{Autor}

La autoría del mayor número de las noticias analizadas pertenece a "Periodistas" $75 \%$ del total (575 noticias). Se refiere en su mayoría a portales de prensa digital. "Otros autores" conforman el 15\% del total (85 noticias). No se especifica su profesión y generalmente suelen ser blogueros. El personal "Sanitario": bien sea médicos, enfermeras, farmacéuticos, psicólogos y/u otros corresponde a un $10 \%$ del total (56 noticias).

\section{Autor}

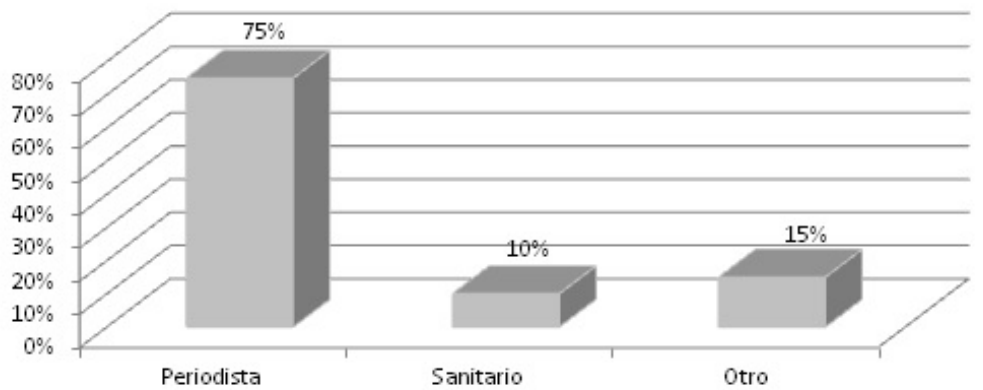

Fuente: Elaboración propia.

\subsection{Valoración del autori sobre el topic}

En este caso la mayoría de los autores ( $72 \%$ del total) de 379 noticias poseen una valoración neutra. En segundo lugar se encuentra la Valoración "Muy buena", correspondiente a un 15\% del total (58 noticias) y "Buena" 9\% (46 noticias).

\section{Valoración del autor sobre el topic}

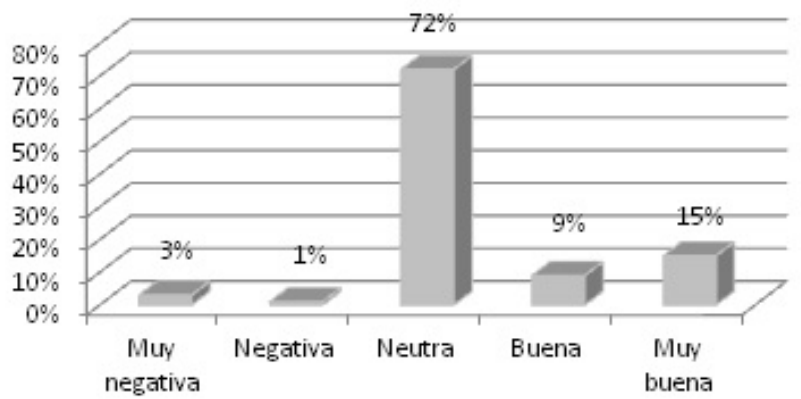

Fuente: Elaboración propia. 


\subsection{Comentarios de los participantes}

Se analizaron un total de 95 debates durante los 11 meses de investigación, pertenecientes a los siguientes sites: prensa digital, blogs serios (blogs pertenecientes a prensa digital), blog catastrofistas (Miguel Jara, Detengan la Vacuna), blog personales (pertenecientes a sanitarios, periodistas, entre otras), Web privadas (Bebes y Más), foro de discusiones (En Femenino, Menéame); entre otros sites.

La mayoría de debates han sido encontrados en páginas catastrofistas o anti vacunas, entre otros portales que proporcionan información negativa sobre las vacunas. En los últimos 4 meses de la investigación surgieron debates en relación a la muerte de una menor por causa de la vacuna VPH (Virus del Papiloma Humano) presuntamente; campaña de vacunación de la gripe y otros temas sobre los que aparecieron posturas negativas al respecto.

Los temas que mayor controversia han provocado han sido los siguientes:

- Evolución de varias enfermedades incluyendo el sarampión en los últimos años.

- Vacunas: ¿obligatorias o no?

- Cada vez son más los padres que no vacunan a sus hijos

- Vacuna del papiloma humano, ¿Un gasto necesario?

5. Comentarios de los participantes

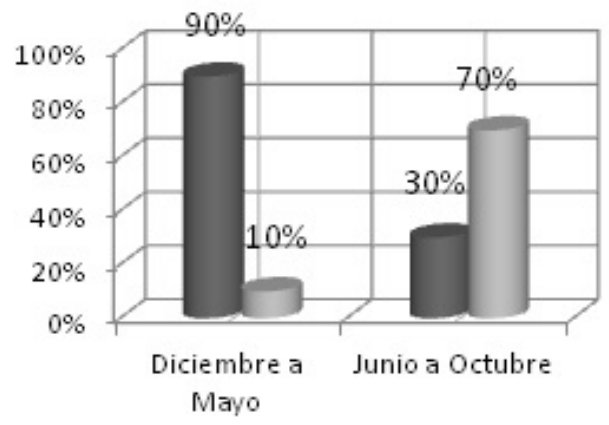

Positivas

Negativas

Fuente: Elaboración propia.

5.6 Rigor de los datos

Se refiere al carácter del rigor de los contenidos encontrado en las noticias y si este contenido es de "base científica" o de "experiencia anecdótica". 
Aparecieron un total de 97\% (700 noticias) con carácter científico: artículos redactados con seriedad, datos científicos e informativos. Por otro lado se contabilizó un $3 \%$ de noticias con carácter anecdótico (16 noticias): artículos redactados bajo el punto de vista del autor sobre determinado tema.

Los canales de comunicación donde se han presentado información anti vacunas han sido Blogs personales, con un $6 \%$ del total de noticias analizadas (44 noticias) y prensa digital especializada en salud con $2 \%$ del total (14 noticias).

Dentro de los portales que ofrecen información anti vacunas, los que mayores datos han aportado son los siguientes:

- www.vacunacionlibre.org: la "Liga para la Libertad de Vacunación" es una asociación plural de ciudadanos (profesionales y usuarios de sanidad), preocupados por la rigidez e indiscriminación de los programas de vacunación. Pretende, entre otros fines, dar a conocer una información libre, contrastada y crítica sobre las vacunas y las consecuencias que provocan.

- www.equipocesca.org: asociación científica sin ánimo de lucro, que busca entender lo que sucede en la consulta del médico general, y su relación con los problemas de salud del paciente.

- www.migueljara.com: web del periodista Miguel Jara donde trata diversos temas, haciendo especial mención a las vacunas y lo perjudicial que pueden llegar a ser en la salud de las personas.

- www.detenganlavacuna.wordpress.com: portal anti vacunas, dedicado a hacer campaña y propagación de mensajes para detener las vacunaciones.

- www.actasanitaria.com: agencia de información especializada en el campo de la sanidad que, a lo largo del tiempo, ha llevado a cabo múltiples actuaciones en su campo específico y, asimismo, ha puesto en marcha y se ha responsabilizado de la edición de diversos medios informativos.

Del total de 716 noticias, tan solo en 57 noticias se han presentado temas relacionados con los portales anti vacunas.

Se realizó un análisis de las 20 noticias con debate en base a la "teoría del frame" (Etman, 1993).

A continuación se presenta un cuadro donde se observan los protagonistas de cada noticia, y a quién se culpa en cada caso respecto al problema o incidencia en el tema de la vacunación. 


\section{Protagonistas de la noticia: "Buenos y Malos"}

\begin{tabular}{|c|c|c|}
\hline \# & Buenos & Malos \\
\hline 1 & $\begin{array}{l}\text { La FDA agencia de medicamentos } \\
\text { estadounidense. }\end{array}$ & Laboratorios Merck \\
\hline 2 & Padres anti vacunas & Doctores \\
\hline 3 & Niños y Padres & Doctores \\
\hline 4 & Familia & Sistema de salud pública \\
\hline 5 & Miguel Jara & Diario el Mundo \\
\hline 6 & Niños y familia & Autoridades médicas, farmacéuticas \\
\hline 7 & Sociedad en general & Farmacéuticas y periodistas. \\
\hline 8 & Médicos en contra de la vacunación & Farmacéuticas y Sanidad pública. \\
\hline 9 & $\begin{array}{l}\text { Afectados de la vacuna del virus del papiloma } \\
\text { humano }\end{array}$ & Farmacéuticas y Sanidad pública. \\
\hline 10 & Personas que reciben las vacunas. & Farmacéuticas \\
\hline 11 & Personas que reciben las vacunas. & Farmacéuticas \\
\hline 12 & $\begin{array}{l}\text { Personas que tienen una postura diferente a la } \\
\text { vacunación }\end{array}$ & Laboratorios Merck \\
\hline 13 & Personas en general & $\begin{array}{l}\text { Centro para el control de prevención } \\
\text { de enfermedades para la vacunación }\end{array}$ \\
\hline 14 & Personas que reciben las vacunas & Farmacéuticas \\
\hline 15 & $\begin{array}{l}\text { Oficina de Evaluación de Medicamentos de } \\
\text { Extremadura }\end{array}$ & Laboratorio Wyeth \\
\hline 16 & Población general. & $\begin{array}{l}\text { Sociedad Española de Medicina } \\
\text { Preventiva, Salud Pública e Higiene } \\
\text { (SEMPSPH) }\end{array}$ \\
\hline 17 & Población general. & Farmacéuticas \\
\hline 18 & Niños y Familia & Sanidad pública y farmacéuticas. \\
\hline 19 & Población en general & Farmacéuticas \\
\hline 20 & Niños y Familia & Farmacéuticas \\
\hline
\end{tabular}

Fuente: Elaboración propia.

Las informaciones de portales anti vacunas posicionan en el papel de "malos" a la "industria farmacéutica" en un 56\%, culpabilizándolos en la mayoría de los casos de hacer negocios con la elaboración de las vacunas.

Les sigue con un $28 \%$ los "doctores/médicos y la Sanidad pública"; en temas relacionados con la vacunación de niños, campañas de vacunación y compra excesiva de vacunas por parte de los gobiernos.

En tercer lugar con un 8\% aparecen los "Medios de comunicación/periodistas" y también "otros". 
Los temas más destacados y debatidos en los sites anti vacunas son: el peligro de "no vacunar" a los niños y la "nueva moda" de no vacunar; reaparición de enfermedades erradicadas en Canarias como consecuencia de la no vacunación; vacunas, anti vacunas, internet y web social; Timerosal y vacunas en la era del autismo, calendario vacunal obligatorio u optativo y laboratorios farmacéuticos llevados a tribunales.

Al tomar una muestra representativa de las noticias y la valoración que realizan los autores sobre los temas, se ha encontrado que un 3\% de las noticias ha sido "muy negativa" y sólo $1 \%$ "negativa". Al verificar la fuente de las noticias se corroboran que pertenecen a portales anti vacunas.

Respecto al rigor de los datos de las noticias sólo un 3\% es de "carácter anecdótico", las afirmaciones que realizan sobre temas de anti vacunación están formadas por estereotipos rígidos, poco rigurosos y carentes de bases científicas.

Durante la investigación (Diciembre-Mayo) han predominado los comentarios positivos hacia la vacunación, no obstante, a partir de Junio-Octubre, comienzan a observarse posturas negativas referentes a las vacunas. Esto se debe a dos aspectos: 1) la mayoría de los debates han sido encontrado en páginas anti vacunas y 2) la muerte de una niña en Asturias relacionada presuntamente con la inoculación de la vacuna del VPH.

Son pocas las páginas anti vacunas presentes en la red, pero éstas están bien organizadas y suelen comentar los mismos usuarios entre una y otra publicación (se hacen "autopromoción" unos de otros). Su presencia en redes sociales no es significativa.

Durante la investigación surgió un suceso que se analizó para verificar la repercusión social obtenida:

- Caso: Muere una Niña en Gijón tras sufrir un ataque de asma severo 10 horas después de haber recibido la vacuna.

Tras el análisis y monitorización diaria de todos los sites citados a lo largo de la investigación se obtuvieron los siguientes resultados:

Tanto en páginas web como en redes sociales se recriminó la vacuna del Papiloma. Personalidades catastrofistas abrieron paso a un gran número de debates negativos; personalidades políticas se pronunciaron en contra de la vacuna. Se produjo una gran confusión en la sociedad con respecto la administración o no de la vacuna.

Se realizó un sondeo sobre todo lo que estaba ocurriendo y se elaboró un informe con todas las noticias pertinentes. Más de 15 diarios fueron eco de la noticia. La web de la Asociación de afectados del Virus del Papiloma Humano hizo seguimiento de la noticia, colocando en su web información de todo tipo (a favor y en contra de la vacuna).

Las autoridades políticas y sanitarias llamaron a la calma y aseguraron investigar el caso hasta el final. 
Tras una búsqueda general en redes sociales sobre la noticia en su mayoría aparecen diarios, personas que reseñan el link de la noticia, pero en ningún caso perfiles de personas relacionadas con los portales anti vacunas.

\section{Conclusiones}

La investigación parece confirmar lo siguiente:

1. Existen reducidos "grupos anti vacunas" que especulan y aportan información negativa sobre las vacunas y cuyas afirmaciones no están basadas en evidencias científicas. Estos grupos parecen retro-alimentarse unos a otros mediante las citas y enlaces entre ellos, pero sin conseguir generar debate entre la población mayoritaria. Tal y como se confirma en los resultados de 716 noticias, tan solo se encontraros 56 noticias relacionadas con el movimiento anti vacunas y solo se encontró debate en 20 de esas noticias. El análisis de las mismas muestra como la información es redifundida y citada por usuarios concretos y pertenecientes al "movimiento anti vacunas" (efecto retro-alimentación).

2. Los topics más importantes analizados en la red sobre el tema anti vacunas son: "Virus del Papiloma Humano o VPH", "gripe", "sarampión", "efectos secundarios de las vacunas", "farmaceúticas" y "brotes de enfermedades", reflejando el interés de los emisores por estos tópicos (más que el interés de los receptores) o población no especializada. Los temas debatidos se repiten con "el peligro de vacunar o no", quejas contra la industria farmacéutica, etc; los cuales siempre se encuentran dentro de una misma línea de debate.

3. El análisis cualitativo en base a la "Teoría del Frame" (Etman, 1993) viene a confirmar que se emplean estereotipos muy rígidos, poco rigurosos y de contenido anecdótico para argumentar en contra de las vacunas. Tiende a clasificarse como "culpables" a la industria farmacéutica de los problemas narradas (daños físicos y muertes, en general). No se plantean con claridad "soluciones al problema" (siguiendo el esquema de Etman). Los contenidos de dichos debates se clasificaron según la "Teoría del Frame" en términos de "buenos" (personas vacunadas y/o afectadas por las vacunas, miembros del colectivo anti vacunas) y "Malos" (industria farmacéutica con un 56\% de las noticias). Tal y como se confirma en los resultados de 716 noticias, tan solo se encontraros 56 noticias relacionadas con el movimiento anti vacunas y solo se encontró debate en 20 de esas noticias. El análisis de las mismas muestra como la información es redifundida y citada por usuarios concretos y pertenecientes al "movimiento anti vacunas" (efecto retro-alimentación). Finalmente se sugiere que la "confianza" en la información presente en la red sobre vacunas es mayor de la que inicialmente podía parecer debido al "aparente ruido" de los "sites anti vacunas" y que éstos no parecen arrojar índices elevados de "confianza en la red", debido a contenidos repetitivos y carentes de rigor. Esta sugerencia 
se basa en el dato anterior: la escasa repercusión de estos sites. Posiblemente esta baja influencia se debe a su baja reputación. Este es, precisamente, el camino que se está explorando actualmente mediante la realización de estudios cualitativos entre la población: mediante el empleo de grupos de discusión se analiza el valor de reputación atribuido a estas páginas. Así mismo, se está desarrollando una metodología cuantitativa para analizar el tiempo y que una muestra de usuarios dedican a navegar por estas páginas así como para medir la valoración reputacional que le atribuyen.

\section{Bibliografía}

BODEMER, N., MÜLLER, S.M., OKAN, Y., GARCIA-RETAMERO, R., NEUMEYER-GROMEN, A. (2012). "Do the media provide transparent health information? A cross-cultural comparison of public information about the HPV vaccine". En Vaccine, n $^{\circ}$ 30, p.3747-3756.

BREIMAN, R.F. (2001). "Vaccines as Tools for Advancing More than Public Health: Perspectives of a Former Director of the National Vaccine Program Office". En Vaccines, $\mathrm{n}^{\circ} 32, \mathrm{p} .283-288$

CHANEL O., LUCHINI S., MASSONI S., VERGNAUD J-C. (2011).'Impact of information on intentions to vaccinate in a potential epidemic: swine-origin Influenza A (H1N1)". En Social Science and Medicine, no 72, p.142-148.

DOWNS J.S., DE BRUIN W.B., FISCHHOFF B. (2008). "Parents vaccination comprehension and decisions". En Vaccine, n 26, p.1595-1607.

EHRETH, J.(2003).'The global value of vaccination, en Vaccine, $\mathrm{n}^{\circ}$ 21, p.596-600.

ENTMAN, R.M. (1993).'Framing: Toward Clarification of a Fractured Paradigm". En Journal of Communication, $\mathrm{n}^{\mathrm{o}} 43, \mathrm{p} .51-58$.

Fundación Farmaindustria (2003). La aportación de las vacunas a la salud. El valor del medicamento.

GUST DA., DARLING N., KENNEDY A., SCHWARTZ, B. (2008). "Parents with doubts about vaccines: which vaccines and reasons why". En Pediatrics, ${ }^{\circ} 4$, p.718-725.

MAYERA, M.A, LEISA, Á. \& SANZ, F.(2009)."Información sobre salud en internet y sellos de confianza como indicadores de calidad; el caso de las vacunas". En Atención Primaria, $\mathrm{n}^{\circ} 10$, p.534-544.

RAO, N; MÖBIUS, M. M., ROSENBLAT, T. (2007)."Social Networks and Vaccination Decisions". En Research Center for Behavioral Economics and Decision-Making, $\mathrm{n}^{\circ} 7$, p.1-50.

STOTO, M.A., EVANS, G., BOSTROM, A. (1998). "Vaccine Risk Communication". En American Journal of Preventive Medicine, nº14, p.237-239.

WATHEN, C.N. \& BURKELL, J. (2002). "Believe it or not: factors influencing credibility on the Web". En Journal of the American Society for Information Science and Technology, $\mathrm{n}^{\circ} 2, \mathrm{p} .134-144$. 
WITTERMAN, H.O \& ZIKMUND-FISHER, B. J. (2011). "The defining characteristics of Web 2.0 and their potential influence in the online vaccination debate". En Vaccine, $\mathrm{n}^{\mathrm{o}}$ 30, p.3734-3740.

\section{Notas}

1 Esta investigación se ha realizado con la colaboración de Sanofi Pasteur MSD y el equipo de investigación del profesor Juan José Picazo (Catedrático de la Facultad de Medicina de la Universidad Complutense de Madrid).

\section{Los autores}

Sandra Gaspar Herrero. Licenciada en Comunicación Audiovisual por la Universidad Complutense de Madrid y Magíster en Gestión Publicitaria. Actualmente se encuentra realizando la tesis doctoral en temas de comunicación y salud en la Facultad de Ciencias de la Información de la UCM. Es becaria de Investigación de la Cátedra de Comunicación y Salud UCM-AbbVie y Colaboradora Honorífica de la UCM. Profesora ayudante de grado de la asignatura "Psicología de la comunicación" y de la asignatura "Comunicación y salud" del Máster Oficial de Comunicación de las Organizaciones, ambos de la Universidad Complutense. Co-directora del Magíster en Comunicación y Salud (UCM-Madrid Salud) y coordinadora académica del Magíster en Gestión Publicitaria (JWT-UCM). Es co-editora de la Revista Científica de Comunicación y Salud.

Ubaldo Cuesta Cambra. Catedrático de la Facultad de Ciencias de la Información de la Universidad Complutense de Madrid, en la cual es Secretario Académico del Departamento de Comunicación Audiovisual y Publicidad II (del cual fue director desde 2004 hasta 2011). Es Director de la Cátedra de Comunicación y Salud de la misma universidad, donde dirige el Magíster en Comunicación y Salud y el Magíster en Gestión Publicitaria (UCM-JWT). Es director del Grupo de Investigación Consolidado de la UCM «Creación y Efectos Psicosociales y Culturales del Discurso Audiovisual», co-director de la Revista Pensar la Publicidad y subdirector de la Revista AdComunica. Ha participado como editor de cinco manuales sobre comunicación y salud, siendo autor a su vez de varios libros y artículos científicos sobre psicología de la comunicación. 